\title{
Menstrual migraines: a case study
}

\begin{abstract}
Migraines are severe headaches that cause debilitating pain. At least half the women that experience migraines report that the occurrence corresponds to their menstrual cycle. This is the case of a 38year-old female with debilitating menstrual migraines lasting thirteen years due to Liver blood deficiency with qi and blood stagnation. After treatment including acupuncture, Chinese herbs, and extensive diet therapy, she has almost completely recovered.
\end{abstract}

Keywords: menstrual migraines, acupuncture, oriental medicine, diet therapy
Volume I Issue 5 - 2015

\author{
Valerie Razutis \\ Southern California University of Health Sciences, USA \\ Correspondence: Valerie Razutis, Southern California \\ University of Health Sciences, 16200 Amber Valley Drive, \\ Whittier, Ca 90604, USA, \\ Email valerie@laughingbuddhaacupuncture.org
}

Received: April 07, 2015 | Published: September 4, 2015

\section{Abbreviations: AOM, acupuncture \& oriental medicine \\ Introduction - biomedicine \\ Migraines affect nearly 28million Americans every year. Although the mechanism is still not fully understood, it is thought that the cause of migraines is sudden vascular dilation in the brain. It is possible this phenomenon is caused by the sudden release of neuropeptides that affect the trigeminal nerve, leading to sudden, painful dilation. ${ }^{1}$ Most of the effective medications for this condition feature at least some sort of vasoconstricting action, giving credence to this theory.}

Patients typically complain of sudden onset moderate to severe pain, usually unilateral in the temporal and parietal regions. In some cases the headache is preceded by an aura similar to that of seizures. ${ }^{1}$ The pain is often accompanied by other neurological symptoms such as sensitivity to light, sound, and odors, as well as nausea and vomiting. For many patients, avoiding known triggers and having access to rapid-acting medications classified as triptans will allow them to successfully avoid debilitating migraines. ${ }^{2}$

Approximately half the women with recurrent migraines report that they occur in concert with their menstrual cycle, typically within a few days of the onset of their menses. ${ }^{3}$ This type of manifestation is known as menstrual migraines. The cause may be related to a sudden drop in estrogen levels just before the onset of menses. Some women begin having migraines at menarche while others see the onset occurring well after puberty, occasionally after an initial pregnancy. The approach to managing menstrual migraines is generally estrogen regulation via oral contraceptives although in many cases this may actually worsen the symptoms. ${ }^{3}$

Diagnosing migraines is based on clinical symptoms. There are no lab or diagnostic tests that confirm migraines. Rather, these tests are used to rule out other identifiable causes for headaches. New onset headaches without an identifiable non-neurological cause should be referred to a neurologist. ${ }^{4}$

Women with menstrual migraines may benefit from prolonged hormonal contraception that reduces menstruation to 2-4 times per year. Many women experience an extreme rebound migraine as a result of estrogen withdrawal when discontinuing the medication. They may also experience an extreme rebound migraine during the placebo period. ${ }^{5}$ These hormonal options carry risks of other side effects unrelated to migraines, such as blood clots and stroke. ${ }^{6}$.For some women, the use of external estrogen actually increases the occurrence and severity of the migraines, both during use and after discontinuation. ${ }^{5}$

In addition to estrogen regulation, patients with recurrent migraines may benefit from the use of pharmaceuticals in the triptan family. These drugs are not analgesics but rather work as serotonin receptor agonists that block the neuropeptides that contribute to the sudden vasodilation and, in reducing vessel size, contribute to pain relief. Other drugs that are found to be helpful include caffeine and ergotamine, both acting as potent vasoconstrictors. Generally speaking, commonly used analgesics such as acetaminophen or NSAIDs are not particularly effective in relieving migraine pain. Opioids, especially when combined with an ergot-derivative are known to be effective but can be habit-forming. The overuse of triptans may cause rebound headaches so patients should be counseled accordingly. ${ }^{1}$

\section{Introduction - acupuncture \& oriental medicine (AOM)}

Migraine is not a specific classification of disease in the classical Chinese texts. Headache is the general term used and can be used to diagnose all of the various causes of headaches, from migraines to tension headaches to common cold headaches. Headache as a disease classification in Chinese medicine is generally used when the headache itself is the primary symptom, as opposed to other conditions where headache is one of many symptoms and not the primary complaint. Migraines typically manifest in patterns of yang rising, either alone or in combination with qi and blood deficiency. ${ }^{7,8}$

In cases with yang rising, there is an identifiable underlying qi stagnation or evidence of underlying qi and blood stagnation that informs the nature of the headache. Patients will complain of acute onset headaches, sometimes preceded by an episode of increased stress. These may be patients with lives colored by high stress. Headaches may be unilateral or bilateral and accompanied by other Liver-Gallbladder symptoms such as bitter taste, irritability, blurred vision, or red eyes. Their pulse may be string-taut and rapid while the tongue may have reddened sides and a yellow coating. Yang rising headaches may be seen in patients with hypertension, tension headaches and cluster headaches as well as migraine headaches. ${ }^{9}$ 
Migraines present typically with rapid onset, unilaterally in the Gallbladder area of the temple and parietal regions of the head, with visual disturbances. This speaks to the Liver yang excess aspect of migraine headaches. The sudden rise of yang through the Gallbladder and Liver channels explains the location and sudden onset. In most cases there are a more complicated excess and deficiency pattern at work, with an underlying deficiency of qi and blood giving opportunity for the sudden yang elevation.

\section{Case history}

The patient is a 38year-old woman that complains of severe, recurrent migraines that occur in the few days directly preceding her menses. Occasionally she will also have a migraine at the time that she ovulates. The migraines began thirteen years ago and have been occurring monthly since then. Three months prior to the onset of the first migraine, the patient underwent a therapeutic abortion at thirteen weeks gestation. She began using hormonal birth control at that time. Just after the procedure she began her current job as a first grade teacher. The patient discontinued the use of hormonal birth control after eight months due to the onset of migraines. She has not used a hormonal contraceptive since then though she continues to have migraines.

The patient reports that her migraines present with generally consistent symptoms. The onset is acute, without aura or visual disturbances. The pain usually starts out as moderate, in the range of 4-6/10. The migraines will gain in intensity over the course of a few hours, reaching $8-10 / 10$. She will occasionally feel nauseous. She can be sensitive to bright lights and sounds during the worst of the pain. She has not found anything that relieves the pain. The pain is described as a throbbing distending pain, particularly worst behind her eye. She does not respond to triptans, caffeine, or ergot-derivative medications. She has tried massage, biofeedback, heat and cold, all without seeing any relief. The migraines typically present on the right side of the head in the temporal and parietal region with referred pain down the side of the neck. Once a migraine starts it will last for 3-5 days. She often has to take at least one day off work during this time.

Her menstrual cycle is very regular at 28-30days. She ovulates regularly at 12-14days. In addition to the migraine, she has limited breast tenderness but no other PMS symptoms. She does not note any cyclical changes in mood and has no food cravings. Her bleeding is scanty. She does not require feminine hygiene products, instead folding a 12-18 inch strip of toilet paper that she changes approximately every two hours, when she urinates. The blood is pale, sometimes watery. There are no clots. She denies any cramping or back pain with her menses.

Prior to the abortion and use of hormonal birth control the patient reports that her bleeding was quite a bit heavier. She used pads and tampons, needing to change them every four hours. Her blood was dark in the first day, gradually becoming lighter. She did not have significant clotting or PMS symptoms, similar to her current menses presentation.

The patient's energy level is low all the time. Her sleep is disturbed by anxiety. She wakes not feeling rested. She generally gets 6-8hours of sleep but wakes early in the morning so the last few hours of sleep are very light and punctuated by frequent waking. Her appetite is good. She eats frequent small meals of generally whole foods. Her diet has less than $1 \%$ animal protein, by her estimation. She does crave red meat at times but tries to avoid eating it. This reduction of animal protein has occurred gradually over the past ten years. She rarely consumes leafy or cruciferous vegetables. She does not plan her diet to consider plant protein levels and does not consume dairy or egg products. Her bowel movements are daily, formed, and do not have a foul odor.

Emotionally, she reports increasing levels of anxiety. She does not feel that she deals with stress or crises well, which affects her performance as a first-grade teacher. She is often lacking in patience when dealing with her students. She has vivid dreams of violent acts. She also reports feelings of grief, depression and sadness. The physical presentation of the patient is very thin. At 5'9" and 125 lbs., the patient's BMI is 18.5 the lowest rating for what is currently considered normal height-weight proportion. Her skin is dry and pale. Her hair is very dry. She reports eye floaters. Her oral and eye mucosa are very pale. Her tongue is very pale, quivering, with deep teeth marks and a thick white coat. There are no sublingual veins. Her pulse is very thin, hard to find in most positions. The Kidney yin pulse in the left chi position is the only strong and easily palpated pulse.

\section{Diagnosis and treatment}

The diagnosis in this case is headaches due to Liver blood deficiency with yang rising in the Gallbladder channel and moderate qi and blood stagnation. Additionally, there is Spleen qi deficiency with blood deficiency. The patient shows signs of long term blood deficiency in her scanty menses, pale dry skin, dry hair, eye floaters, and fatigue. Her pale quivering tongue with deep tooth marks demonstrates blood and qi deficiency, confirmed by her very thin and hard to palpate pulses. Her chronic fatigue, lack of restful sleep, and depression point to long-term qi deficiency. Her diet of whole foods is generally healthy but lacking in sufficient animal protein and iron-rich vegetables. The blood deficiency allows for space to exist within the vessels, allowing the unanchored yang to ascend rapidly, causing severe headaches. The location of the migraines speaks to the Gallbladder involvement in her case.

The patient has qi and blood stagnation that we see manifesting as depression. Her work with small children is quite stressful. These emotional strains contribute to qi stagnation, giving further opportunity for space in the vessels allowing yang to rise. The very specifically gynecological nature of her migraines points directly to the Liver and Liver qi stagnation as a primary contributor to her condition.

The treatment principle is to tonify Liver blood, smooth the flow of Liver Qi, anchor the yang, stop pain, and promote menses. This is managed with acupuncture, Chinese herbs, and nutritional therapy. The patient received acupuncture treatments scheduled every two weeks to occur just prior to ovulation and menstruation. Acupuncture points were selected to tonify Liver blood, tonify the Spleen, gently move qi and blood, and calm the shen. The points used include Du20, Sp6, St36, P6, Sp4, GB41, LI4, Liv3, and the extra pointM-HN-20.

For most treatments the patient received Du20 to subdue the yang, as well as Sp6, St36 to strongly tonify blood and qi. P6 and Sp4 were used to open and activate the Chong channel, to allow it to fill with blood and promote adequate menses. LI4 and Liv3 gently course the Liver qi, helping to reduce stagnation and relieve pain. GB41 is used for the special function of relieving headaches, particularly one-sided headaches. In this case, it is needled on the right side, as that is the side where the migraines occur most often. The patient consistently 
reported significantly greater point tenderness on the right as compared to the left. Additionally, GB41 opens the chest and relieves binding in the chest. ${ }^{10}$ When combined with $\mathrm{P} 6$ this helps to unbind the emotional constraint and allow the patient to process past emotional pain and loss.M-HN-20 was added to the prescription to calm the she and reduce stress. The patient reported feeling calmer after treatments and more at peace with herself. This would be followed by one to two days of intermittently feeling sadness and grief but not a debilitating sadness as she had felt prior to beginning treatment.
For the herbal approach, a custom powdered formula was created combining the ingredients of Xiao Yao San adding Shu Di Huang and ChuanXiong, the two ingredients of Si Wu Tang that are not already contained within Xiao Yao San. The formula is presented as follows:

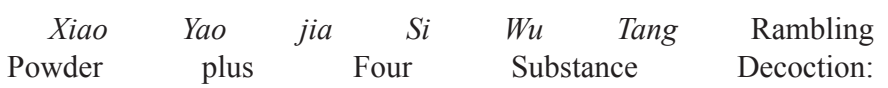

Two parts

\begin{tabular}{llll}
\hline Two parts: & & \\
\hline Dang Gui & Radix angelicae sinensis & Angelica sinensis & root \\
Bai Zhu & Rhizoma atractylodis & Atractylodes macrocephala & root \\
Bai Shao & Radix paeoniae alba & Paeonia suffruticosa & \\
Fu Ling & Poria & Wolfiporia extensa & root fungus \\
Chai Hu & Radix bupleuri & Bupleurum falcatum & root \\
Shu Di Huang & Radix rhemanniae praeparata & Rehmannia glutinosa & root: steamed \& dried \\
Chuan Xiong & Rhizoma chuanxiong & Ligusticum wallichii & root \\
Sheng Jiang & Rhizoma zingiberis recens & Zingiber officinale & root \\
One part: & & & \\
Bo He & Herba menthae & Mentha haplocalyx & stem \& leaves \\
Zhi Gan Cao & Rhizoma glycyrrhizae praeparata Glycyrrhiza glabra & honey fried licorice root \\
\hline
\end{tabular}

The purpose of this formula was to strongly tonify blood along with gentle moving to prevent further stagnation. The formula was prescribed as a 5:1 granular concentrate with a dosage of four grams twice daily in hot water. The patient was given extensive diet therapy. She did not have strong feelings regarding eating meat but rather migrated towards a purely plant-based diet because she felt she did not digest meats well. She reported fatigue and feelings of heaviness after eating beef and pork but stated that when she ate meat regularly her diet was primarily animal-based, with minimal vegetable consumption. She was advised to significantly increase her consumption of dark leafy greens such as spinach and kale. She was given recipes for both beef and chicken bone soup and advised to consume small quantities of fish and lean chicken or beef, increasing her protein intake to $20-30 \%$ of her total daily intake. ${ }^{11}$

After two months of treatment the patient reported significant improvement in the migraines. In the first month after beginning treatment, she did develop a migraine prior to her menses but the pain did not exceed 3/10 and lasted only one day. In the second month she experienced only a brief period of mild aching lasting a few hours that did not require medication. Her menses did not change significantly. Her bleeding continued to be scanty. The patient acknowledged that she struggled to be compliant with the herbal formula and diet therapy. She was able to drink the herbal tea only once every other day rather than the twice daily dosage prescribed. She stated that this was due to her schedule and that she found drinking tea to be inconvenient for her. She requested a change to a pill formula.

She noted that whenever she made bone broth she consumed it immediately as if she was "starving" and felt much more energetic for a few days but that she could not find the time to make large quantities of broth more than once or twice per month. To address this, the patient was given instructions on the use of a crockpot that she could use to cook broth several times per week with minimal effort. She was given recipes for several soups using bone broth that freeze well and for ways to incorporate bone broth in place of water, allowing her greater flexibility in the use of the broth and greater variety in her food intake. ${ }^{12}$

There is no custom patent pill available that combines xiaoyaowan and siwutang. The powdered herbal prescription was replaced with Health Concerns Chong Release Formula, a modified taohongsiwutang. This formula was available as water decocted concentrate at 7.5:1. The patient was prescribed three pills twice daily. This formula is designed to strongly tonify blood with moderate blood moving to prevent stagnation. It also supports the qi and calms the shen.

After the fourth month of treatment, eight acupuncture treatments total, the patient reported that she had not had a migraine in either of the preceding menstrual cycles. She was able to be much more compliant with the herbal formula in pill form but still missed several doses each week. She also improved her diet considerably, making a much greater effort at home cooking, using the soup recipes she was given and cooking with friends. Her menses increased in volume to a degree that required the use of low-absorption panty liners. She reported that the bleeding was still pale and considerably less in volume than her pre-complaint menses. The patient was referred to her primary care physician for a complete blood count to rule out anemia but she was reluctant to follow through on that referral. She also declined to follow through on lab tests ordered by the acupuncturist. She added a liquid iron supplement and a complex B vitamin supplement to her diet and reported significant increases in energy as well as moderate increase in the volume of bleeding during her menses.

\section{Discussion}

Through the use of acupuncture, Chinese herbs, and Diet therapy, this patient has improved significantly. Continued acupuncture to manage stress is recommended to complement the coping mechanisms she has learned in talk therapy.

Compliance with treatment may continue to be a challenge for this patient and may deter full recovery. She has thus far responded well 
to treatment. Her headaches have diminished significantly while her menstrual flow has improved slightly. There is still much progress to be made to return her to full health. To do this, she will need to commit herself to eating well, resting well, controlling her stress, and continuing with regular acupuncture treatment. If she can successfully manage the first three of those needs, she can see needed acupuncture treatments reduced to monthly or even quarterly.

\section{Acknowledgments}

None.

\section{Conflict of interest}

The authors declare that there is no conflict of interest.

\section{Funding}

None.

\section{References}

1. Porter RS. The Merck Manual of Diagnosis and Therapy. 19th edn, USA: Merck; 2011.

2. Longo D, Fauci A, Kasper D, et al. Harrison's Principles of Internal Medicine, $18^{\text {th }}$ edn, USA: McGraw-Hill Professional; 2011.
3. MacGregor EA. Menstrual Migraine: Therapeutic Approaches. Ther Adv Neurol Disord. 2009;2(5):327-336.

4. McPhee S, Papadakis M, Rabow MW. Current Medical Diagnosis and Treatment 2011. 50 ${ }^{\text {th }}$ edn, McGraw-Hill Medical.

5. Karch AM. Lippincott's Nursing Drug Guide 2013. 1st edn., USA: Lippincott Williams \& Wilkins; 2012.

6. PDR Staff. Physicians' Desk Reference 2013. USA: PDR Network; 2013.

7. Xinnong C. Chinese Acupuncture and Moxibustion. 3rd edn. China: Foreign Languages Press; 2010.

8. Maclean W, Lyttleton J. Clinical Handbook of Internal Medicine. 1st edn. USA: Redwing Book Co; 2010.

9. Maciocia G. Diagnosis in Chinese Medicine: A Comprehensive Guide. 1st edn. USA: Churchill Livingstone; 2004.

10. Deadman P, Al-Khafaji M, Baker K. A Manual of Acupuncture. 2nd edn. Journal of Chinese Medicine Publications.

11. Pitchford P. Healing with Whole Foods: Asian Traditions and Modern Nutrition. 3 Rev Upd. USA: North Atlantic Books; 2003.

12. Bliss N. Real Food All Year: Eating Seasonal Whole Foods for Optimal Health and All-Day Energy. 1st edn, USA: New Harbinger Publications; 2012. p. 192. 